(Aus dem physiologischen Institut der Universität Wien.)

\title{
Beiträge zur Kenntnis des Druckphosphens. Von
}

\section{Dr. Robert Stigler,}

d. Z. Assistent am physiologischen Institut der Universität Graz.

(Mit 3 Textfiguren.)

Das Druckphosphen war schon Aristoteles bekannt. In der Neuzeit findet sich seine Beobachtung zuerst bei Newton ${ }^{1}$ ), D. Jurin ${ }^{2}$ ), Eichel ${ }^{3}$ ) und J. Elliot ${ }^{4}$ ); eingehend hat sich Purkinje ${ }^{5}$ ) mit derselben befasst. Der Name "Phosphen" für das Bild der mechanischen Reizung der Netzhautperipherie findet sich zuerst bei Serre d' $U z \mathrm{ès}^{6}$ ).

Die Beschreibungen, welche die Autoren vom Druckphosphen geben, weichen nicht unerheblich voneinander ab. Elliot vergleicht es mit einem Pfauenauge (Auge einer Pfauenfeder). Über die Farbe derselben schreibt er: "Nach anderen Beobachtungen ist auch der Ring oder Fleck, der bei dem Druck des Augenwinkels entsteht, zuweilen verschieden gefärbt. Erscheint der Ring sehr hell, so fällt er ins Gelbliche oder Rötliche, ist er aber blässer, so sieht er vielmehr grünlichblau aus."

Diese Beobachtung ist später von $\mathrm{Nag} \mathrm{el}^{7}$ ) bestätigt und auf

1) Optice, Quaestio vol. XVI. 1706.

2) D. Jurin, Essay upon distinct and indistinct Vision in Schmith optics Cambridge 1738.

3) Eiche 1, Experim. circa sensum videndi. Collectan. societ. med. Havniensis vol. 1.1774.

4) J. Elliot's physiologische Beobachtungen über die Sinne, besonders über das Gesicht, das Gehör, sowie auch das Brennen und die tierische Wärme. Leipzig 1785.

5) Purkinje, Beobachtungen und Versuche zur Physiologie der Sinne Bd. 1 S. 128 , I. XXIV.

6) Essai sur les Phosphènes etc. Paris 1853. Vic. Masson. Compt. rend. t. 31 p. $375-378$.

7) W. A. Nagel, Einige Beobachtungen über die Wirkung des Druckes und des galvanischen Stromes auf das dunkeladaptierte Auge. Zeitschr. f. Psych. u. Phys. d. Sinnesorgane Bd. 34 S. 285. 
den Adaptationszustand des Auges zurückgeführt worden. Nagel erschien das Druckphosphen im dunkeladaptierten Auge bläulichweiss, im hell adaptierten rötlichgelb.

Ausserdem beschrieb Elliot die Veränderung des Phosphens bei Drucksteigerung:

"Drücke ich den äusseren Augenwinkel stärker als gewöbnlich, so erhalte ich zuweilen zwei Ringe, auf jeder Seite des Gesichtes einen, davon der auf der entgegengesetzten Seite des gedrückten Winkels auf gewöhnliche Art, der andere aber, wie ich glaube, daher entstand, dass sich der Druck gerade durch den Durchmesser des Augapfels fortpflanzte und die Feuchtigkeiten gegen die entgegengesetzte Seite der Netzhaut vordrückte." Hielt er mit dem Seitendruck an und verstärkte ihn immer mehr, so bemerkte Elliot, dass zuerst der Lichtring verschwand und schliesslich sich ein gleiches „allgemeines Licht" zeigte, wie wenn man sich mit dem Ballen der Hand auf das Auge drückt.

Diesen letzteren Versuch fand aber Elli ot so schmerzhaft, dass er ihn für gefährlich für das Auge hielt. Purkinje's Beobachtungen muss ich wohl ausführlich wiedergeben, erstens weil eine willkürliche Kürzung deren Beschreibung unverständlich machen würde, und zweitens weil seine Schriften wohl nur sehr schwer zu erhalten sind.

Purkinje fand, dass bei Akkomodation für die Nähe schon die leiseste Berührung diese „feurigen Ringe“, wie er das Druckphosphen nennt, hervorrufe. Er erklärte sie durch Zerrung der Retina. Wenn er ein weisses Papier an den inneren Augenwinkel hielt und das Auge stark nach innen wendete und dann am äusseren Augenwinkel mit einem stumpf zugespitzten Hölzchen einen Druck tief gegen die Orbita durch das Augenlid ausübte, so sah er auf dem vorgehaltenen weissen Papier einen grossen, annähernd kreisförmigen Fleck ${ }^{1}$ ); an der dem Fixationspunkte zugewendeten Seite desselben sah er viele parallele, konzentrische, abwechselnd schwarze und weisse bogenförmige Linien, die nach innen immer kürzer werden. Den entgegengesetzten Rand des Fleckes fand Purkinje ${ }^{2}$ ) "mit gelblichweissem Lichte begrenzt, das bei vermehrtem Drucke mit dem Stiele bald sich gegen die Mitte verbreitet, bald den Fleck in zwei Teile scheidet. Der Fleck selbst ist an

1) Siehe Purkinje, Beobachtungen und Versuche, I Fig. 29.

2) Beobachtungen und Versuche, I S. $140 \mathrm{ff}$. 
der Peripherie dunkelschwarz, nach innen dunkelblaugrün, auch dunkelviolett schillernd, was bei wohlbedecktem Auge ein schwaches Schimmerlicht gibt. Mit Recht vergleicht ihn Elli ot mit dem Auge einer Pfauenfeder. In ihm bemerkt man mehrere Partien der oben beschriebenen Aderfigur schwarz in dem schillernden Lichte des Pfauenauges, die gerade dieselbe Verästelung haben wie dort angegeben ist."

„5. Wenn man sehr weit nach hinten drückt, dass die parallelen Streifen bis in die Mitte des Gesichtsfeldes gehen, so zeigen sich hier zwei weisse Bänder, die sich verbreiternd unter einem stumpfen Winkel nach aussen gehen und zwischen sich einen kleinen lichten Fleck haben; an der andereu Seite ihrer Vereinigung ist ein bräunlicher halbmondförmiger Fleck. Beide folgen den jedesmaligen Bewegungen des Pfauenauges, indem sie sich um den Mittelpunkt des Sehfeldes wie um eine Achse drehen. Wurde der Druck verstärkt, so rückt der schwarze Fleck bis gegen die Mitte hin, verschlingt die Schenkel der Bänder bis auf die Stelle ihrer Vereinigung, die sich nun als ein weisser kreisrunder Fleck darstellt. Der halbmondförmige Fleck macht sich auf, legt sich zurück und verschwindet in den hinter ihm gelegenen Parallelen, indes ein halbkreisförmiger Vorsprung aus dem übrigen Lichte hineinrückt."

„6. Wenn man von dem Drucke an der Seite des Augapfels plötzlich nachlässt, so zieht sich der weisse Kreisfleck ebenso schnell nach aussen zuruck, und an seiner Stelle bleibt eine kurze Zeit ein leicht braunvioletter Nebel, der durch einen weissen Streifen in zwei Teile geteilt ist"...., Manchmal bleibt er, besonders in der Mitte, längere Zeit zurück und hindert das deutliche Sehen . . . .

„8. Bei verfinstertem Gesichtsfelde erscheinen die beschriebenen schillernden Farben in der Mitte des Kreisfeldes leuchtend, sowie auch der äussere Rand, der den schwarzen Ring umgibt; die konzentrischen Striche sind nicht unterscheidbar und geben nur einen matten Schein. Wenn man schnell vom Drucke nachlässt, so bewegt sich jedesmal ein heller Lichtstreifen von innen nach aussen gleich einem Blitzstrahle. Das bei offenem Auge gesehene Gelblichweiss am äusseren ${ }^{1}$ ) Rande des Kreisfleckes ist bei bedecktem schwarz und reicht ebenfalls gegen die Mitte des Fleckes hinein. Es ist also

1) Gemeint ist von dem Mittelpunkt des Gesichtsfeldes aus, also am peripheren Rand. 
ursprünglich durchsichtig und hier nur schwarz aus Mangel des äusseren Lichtes. Dagegen ist das Schwarz des Randes und der konzentrischen Linien eine wirkliche Sensation und behauptet sich auch gegen das äussere Licht." Ausserdem sah Purkinje bei trillerndem Drucke auf den äusseren Augenwinkel des nach innen gewendeten Auges einen Lichtkreis in der Gegend der Papille von etwa $30^{0}$ Durchmesser ${ }^{1}$ ).

$A u b$ ert $t^{2}$ ) wiederholte diesen Versuch und beschrieb seine Beobachtung wie folgt:

„Wenn ich im Hellen nach Purkinje's Anweisung in den inneren Augenwinkel die Ecke eines weissen Papierkartons bringe, das Auge stark nach innen wende und an der äusseren Seite des Bulbus mit einem Stecknadelkopf drücke, so sehe ich, auf das Papier projiziert, an der Nasenseite einen dunklen Fleck mit heller Einfassung. Der dunkle Fleck ist etwa birnförmig mit der Spitze nach dem fixierten Punkt gerichtet; die Gegend des fixierten Punktes erscheint als ein mattgrauer Fleck, und endlich erscheint ein grauer unbestimmter Fleck mit hellem Rand, der Gegend des blinden Fleckes entsprechend. Lasse ich mit dem Drucke plötzlich nach, so erscheint an der fixierten Stelle ein heller Fleck, die beiden anderen verschwinden, ohne dass ich eine Umkehrung der Erscheinung wahrnehmen kann."

Helmholtz $z^{3}$ bildet das Druckphosphen ab, wie es ihm auf einem gegen den inneren Augenwinkel gehaltenen Papierblatt erscheint. Es „besteht aus einem dunklen Fleck, von einem hellen senkrechten Streifen durchzogen. Von dem dunklen Flecke geht, wenn man in richtiger Höhe drückt, ein horizontaler Fortsatz aus, dessen Spitze ... den Fixationspunkt berührt, und ausserdem ist in der Gegend des Sehnerveneintrittes ein unbestimmt gezeichneter Schatten ... sichtbar". Das System feiner, paralleler, bogenförmiger Linien zwischen dem dunklen Druckbilde und dem Fixirpunkte sah er nicht so ausgebildet wie Purkinje, am besten bei grosser Helligkeit der entsprechenden Stelle des Gesichtsfeldes. „Im dunklen Gesichtsfelde dagegen erscheint eine helle gelbliche Kreisfläche, in deren Innerem sich zuweilen ein dunkler Fleck oder ein dunkler

1) Purkinje, Beobachtungen und Versuche, II S. $113 \mathrm{ff} .1825$.

2) Aubert, Physiologie der Netzhaut, V. Kap., S. 330. Breslau 1865.

3) Handb. d. phys. Optik, 2. Aufl, S. 236 u. Taf. I Fig. 8. 
Ring abzeichnet. Ein schwaches Licht erscheint auch an der Eintrittsstelle des Sehnerven".... Nur den Fortsatz zum gelben Fleck hin hat $\mathrm{Helmholtz}$ im dunklen Felde nicht sehen können.

Auch Reich ${ }^{1}$ ) sah unter den gleichen Bedingungen einen dunklen Fleck am Fixationspunkt und nach innen davon einen zweiten dunklen Fleck.

$\mathrm{Fick}^{2}$ ) sah "bei geschlossenem Auge im dunkeln Gesichtsfelde eine lichte Scheibe mit dunklem Rande, der noch einmal von einem hellen umsäumt ist". . . „Macht man den beschriebenen Versuch bei offenem Auge, so zeichnet sich das Phosphen im ganzen dunkel im hellen Gesichtsfelde. Daraus ist zu schliessen, dass der Druck die von ihm getroffenen Netzhautelemente nicht nur reizt, sondern auch ihre Reizbarkeit für Strahlung herabsetzt."

Als helles Zentrum, umgeben von einem dunklen und einem hellen Kreis, beschreiben das Phosphen J. Ranke ${ }^{3}$ ) und H. Beaunis ${ }^{4}$ ).

E. Fu chs $^{5}$ ) schreibt, dass er die von Purkinje beschriebenen konzentrischen Streifen am Ranue des Druckphosphens sehr deutlich sehe.

G. Schwarz ${ }^{6}$ ) bemerkt folgendes: Geringer Druck mit dem Stecknadelkopf am äusseren Augenwinkel bleibt wirkungslos, bei stärkerem Druck taucht ein leuchtender runder Fleck auf, bei sukzessiver Drucksteigerung eine dunkle, von einem hellen Rand umgebene Scheibe, bis auch diese erlischt; darin erblickt er das Gesetz von der Schwelle, der erregenden und lähmenden Wirkung des Reizes, indem die Lähmung von der Stelle des höchsten Druckes, dem Zentrum, nach der Peripherie fortschreitet.

Fr. Klein schreibt ${ }^{7}$ ): „Bei verdunkeltem Auge ist der Fleck hell, scharfrandig, die Randpartien meist heller als die oft etwas wolkige Mitte. Bei offenen Augen am Tage sehe ich ihn ebenso gross ...., aber dunkel (gewöhnlich mit einem der Aufmerksamkeit leicht ent-

1) Reich, Über einige subjektive Gesịchtserscheinungen bei gesteigertem intraokulären Druck. Klin. Monatsbl. f. Augenheilk. 1874 S. 238.

2) In Hermann's Handb. d. Physiol. Bd. 3 (1) S. 228. 1879.

3) Grundzüge der Physiologie des Menschen. 1872.

4) Nouveaux éléments de Physiol. humaine p. 1138. Paris 1881.

5) E. Fuchs, Über eine entoptische Erscheinung bei Bewegung des Augapfels. Gräfe's Arch. f. Ophthalm. Bd. 27 S. 3. 1881.

6) G. Schwarz, Beobachtungen bei der mechanischen Reizung der Netzhaut. Zeitschr. f. allgem. Physiol. Bd. 3 (2) S. 89. 1903.

7) Das Wesen des Reizes. II. Arch. f. Anat. u. Physiol. 1905 S. 149. 
gehenden äusserst schmalen helleren Rande").... „Das Eigenlicht der Netzhaut ist weiss (höchstens etwas gelblich); es wird also auch weisses Licht absorbieren. Daber erscheint die durch Druck zum Selbstleuchten gebrachte Netzhautstelle dunkel, wenn helles weisses Licht ins Auge gelangt. Die leuchtende Netzhautschicht muss zwischen äusserer Lichtquelle und Stäbchen- und Zapfenschicht (also nach innen von dieser Schicht) liegen."

Trotz der eingehenden Beobachtungen, welche über dieses Phänomen bereits publiziert worden sind, scheint es mir nicht überffüssig, denselben noch die meinigen anzureiben, welche ich seit $21 / 2$ Jahren in ungezähiten Versuchen gemacht habe, und zwar einerseits um der Beobachtungen selbst willen, weJche ja wie bei allen subjektiven Gesichtserscheinungen von individueller Veranlagung und persönlicher Übung in hohem Grade abhängig sind, anderseits wegen der Folgerungen, welche daraus für die Theorie des Sehens gezogen werden können. Sowohl die Beobachtungen über das Druckphosphen selbst als auch die daraus gezogenen Schlüsse sind, wie schon die kurze vorausgehende Literaturübersicht lehrt, bei den einzelnen Autoren vielfach widersprechend.

Da das Druckphosphen der Fovea am nächsten gebracht wird, wern das Ange möglichst weit med̉al gewendet und dann im lateralen Augenwinkel gedrückt wird, so habe ich diese Stellung bei meinen Versuchen eingehalten. Ausserdem aber wurden dreierleí verschiedene Versuchsbedingungen gewählt:

I. bei offenem Auge im Hellen;

II. bei offenem oder geschlossenem Auge im Dunkeln;

III. bei geschlossenem Auge im Hellen, wobei das Licht durch die Lider fällt.

\section{Versuch bei offenem Ange im Hellen.}

Ich wende das zu drückende Auge medial, so dass es auf den Nasenrücken blickt, und drücke nun mit dem Nagel des kleinen Fingers im lateralen Augenwinkel gegen den Bulbus. Das entstehende Phosphen projiziere ich, soweit es geht, auf meinen Nasenrücken, welcher von einer Lichtquelle her mittelmässig beleuchtet ist.

Drücke ich nur ganz gelinde, so sehe ich zuerst einen hellen Fleck am Nasenrücken, sowie dies auch Schwarz (s. o.) beschreibt. 
Bei stärkerem Drucke erblicke ich das in Fig. 1 abgebildete Phänomen, welches aus folgenden Teilen besteht:

1. Eine Figur aus konzentrischen Kreisen, mit einem hellen runden Zentrum von gelblichweisser Farbe, umgeben von einem breiteren schwarzen Ringe, welcher wieder von einem ziemlich scharf begrenzten, äusserst deutlichen weissgelben Ring umschlossen ist; an diesen schliesst sich aussen wieder ein dunkler Ring an, der unscharf begrenzt in den übrigen Hintergrund übergeht.

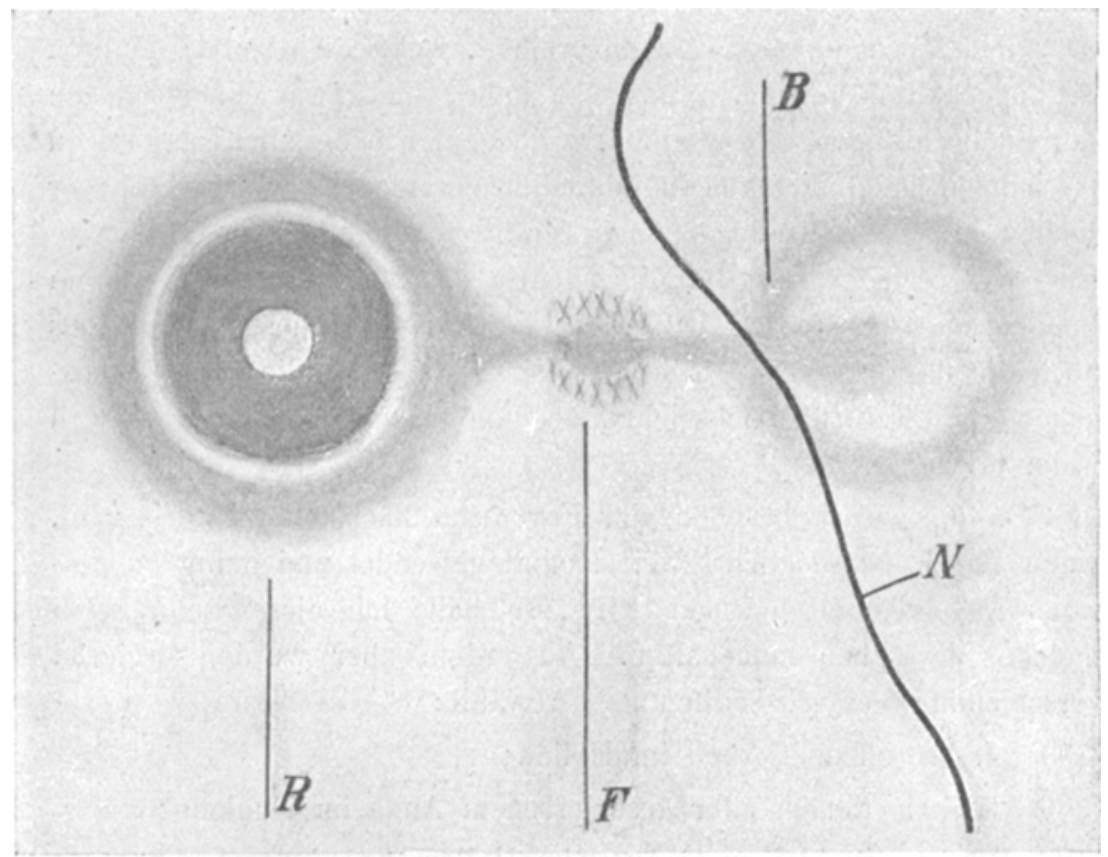

Fig. 1. Das Druckphosphen, wie ich es bei mittelstarkem Druck auf den Bulbus im Hellen sehe. Bei Druckänderungen treten Veränderungen in der Grösse und Umwandlungen der Kreise zu Ellipsen oder Parabein auf. $-R$ Ringfigur, der gedrückten Stelle entsprechend. $F$ Fixationspunkt; bei trillerndem. Drucke von einem zarten Netzwerk umgeben. $B$ Blinder Fleck. $N$ Nasenrücken.

2. Von dieser Ringfigur erstreckt sich gegen den Fixationspunkt ein dunkler zapfenförmiger Fortsatz, â der im Fixationspunkte selbst knopfartig endigt; diese dunkle Stelle ist wieder von einem matten Hofe umgehen, in welchem sich ein feines Karree zeigt, wie eine kleine Schachfigur, von abwechselnd hellen und dunklen Vierecken, wenn man einen intermittierenden Druck auf den Bulbus ausübt.

3. Vom dunklen Fixationspunkte geht wieder eine matte Garbe 
gegen den blinden Fleck $\mathbf{z u}$, an dessen äusserer Seite ein runder Schatten erscheint, der von einem breiten hellen Kreisring umgeben ist; letzterer geht durch den blinden Fleck und erseheint nur an dessen Stelle unterbrochen. Dieses runde Phosphen an der äusseren Seite des blinden Fleckes ist fast ebenso gross wie die Ringfigur, welche der gedrückten Stelle entspricht. Über seine Lagebeziehung zum blinden Flecke orientierte ich mich durch gleichzeitige genaue Umgrenzung des letzteren.

Die Ringfigur an der äusseren Seite des blinden Fleckes tritt bereits bei blosser Einwärtswendung des Bulbus auf und wurde zuerst von Purkinje ${ }^{1}$, dann von $\mathrm{Czermak}^{2}$ ), Aubert ${ }^{3}$ ) und Helmholtz $z^{4}$ beschrieben. Jedoch meinte Purkinje, dass sie in toto dem blinden Fleck entspräche. Dass dies aber nicht so ist, hat Helmholtz durch eigene Beobachtungen und Versuche erwiesen ${ }^{5}$ ).

Durch den Druck auf den Bulbus wird der bei der blossen Einwärtswendung desselben auftretende viel schmälere helle Ring mit seinem grossen runden dunklen Zentrum verbreitert und demgemäss das dunklere Zentrum verkleinert.

Übt man den Druck im lateralen Augenwinkel bei geradeaus gerichtetem Blicke aus, so ist das Phänomen in der Gegend des blinden Fleckes sehr undeutlich, und es lässt sich daran nur eine Helligkeit mit mattem Zentrum erkennen, ohne dass es mir möglich wäre, ein klares Bild hiervon zu bekommen.

Am besten kann man dieses Phänomen auf einem mattgrauen Hintergrund beobachten; sehr günstig fand ich es, diesen dadurch zu erzeugen, dass man nach einem vor eine Lichtquelle (bedeckten Himmel) gehaltenen Milehglas blickt und das Phänomen somit auf das Milchglas projiziert.

Wenn ich, während dieses Phänomen bei möglichst konstantem Drucke besteht, ruhig fixiere, so verschwindet zuerst das zartere Bild des Sehnerveneintrittes, dann der dem Fixationspunkte entsprechende Teil der Figur mit den zu ihm ziehenden dunklen Dreiecken, dann das helle Zentrum der Kreisfigur, dann deren heller Rand, dann endlich der dunkle Kreisring selbst.

1) Beobachtungen und Versuche, I, Kap. X, S. 79.

2) Czermak, Physiol. Studien. 1854.

3) A ubert, Physiologie der Netzhaut S. 338.

4) Helmholtz, Physiol. Optik, Taf. V. Fig. 2.

5) Physiol. Optik, II. Aufl., S. 726.

E. Pflüger, Archiv für Physiologie. Bd. 115. 
Höre ich jetzt oder auch schon früher, nach etwa 2-3 Sekunden, mit dem Fingerdruck plötzlich auf, wobei ich in der gleichen Richtung möglichst ruhig weiterfixiere, so tritt eine ausserordentlich deutliche Umkehr aller Helligkeitsverhältnisse auf, und zwar in der Gegend des Fixationspunktes mit längerem Bestande, in der Peripherie aber nur blitzartig, was die Beobachtung derart erschwert, dass ich mir davon erst nach sehr vielen Versuchen ein klares Bild machen konnte. An Stelle des schwarzen Ringes zeigt sich ein sehr heller Ring mit einem wegen der peripheren Lage am schwersten zu. beobachtenden dunklen Zentrum; dieser ist von einem sehr deutlichen schwarzen Kreis umgeben, von welchem wieder an Stelle des früher dunklen ein nun gelblichgrün leuchtender Zapfen gegen den Fixationspunkt zieht. Dieser selbst zeigt sich mit grösster Helligkeit als gelblichgrüner Fleck am Nasenrücken und verschwindet erst nach etwa $1-2$ Sekunden ${ }^{1}$ ).

In der Gegend des blinden Fleckes blieb ein sehr deutlicher dunkler Kreis bestehen, jedoch nicht lange. Zugleich sah ich dort bäufig beim Aufhören des Druckes die Schatten der Retinalgefüsse gemäss dem plötzlichen Einschiessen des Blutes in die Venen auftreten.

\section{Versuche im Dunkeln.}

Stelle ich den im vorigen Absatz beschriebenen Versuch im Dunkeln, bei offenem oder geschlossenem, nach einwärts gewendetem Auge an, so sehe ich die Kreisfigur ganz in der gleichen Art wie im Hellen: ein leuchtendes Zentrum, umgeben von einem breiten schwarzen Ring, an den sich wieder ein leuchtend weisser Ring anschliesst. Der Unterschied gegenüber der Ringfigur im Hellen besteht nur in dem mondlichtartigen Glanze der lichten Teile. Ich muss diesen auch von meinen Kontrollpersonen angegebenen Befund besonders betonen, da sich in der Literatur gegenteilige Beobachtungen finden, aus denen sogar wichtige Folgerungen gezogen wurden.

Der deutlichste Beweis davon, dass das Druckphänomen auf dunklem Grunde ebenso erseheint wie auf hellem, wird, wie mir Herr Hofrat S. Exner freundlichst vorschlug, erbracht, indem man einen zur Hälfte schwarzen und zur Hälfte weissen Papierstreifen an

1) Die grüne Nuance ist wohl durch den Kontrast mit der rötlichen Hant des Nasenrückens bedingt. Die Umkehr der Helligkeitsverhältnisse des Ringphosphens zeigte sich besonders deutlich, wemn ich den Versuch nachts in Lichte einer Bogenlampe anstellte. 
den Nasenrücken im inneren Augenwinkel derart anlegt, dass sich das durch Druck des Fingernagels erzeugte Phosphen zur Hälfte auf Schwarz, zur Hälfte auf Weiss projiziert. Es erscheint dann die eine Hälfte des schwarzen Ringes auf dem schwarzen Grunde dunkler als dieser, während der jenen umgebende weisse Kreisring mit seiner auf den hellen Grund fallenden Hälfte diesen an Helligkeit deutlich übertrifft.

Übe ich am lateralen Bulbusteil wieder einen intermittierenden Druck aus, so erscheint mir ausserdem in der Gegend des blinden Fleckes ein schwach heller Kreis und im Fixationspunkt ein bläulicher Schimmer, von welchem eine gleich schimmernde Brücke sowohl zur Ringfigur als auch zum blinden Fleck zieht.

Bei ruhigem Drucke erscheint aber nur die Ringfigur deutlich. Unterbreche ich diesen Druck plötzlich, so blitzt die Ringfigur hell auf, während sich in der Gegend des blinden Fleckes ein sehr dunkler Kreis zeigt, der von einem hellen Ring umgeben scheint. Einè deutliche Umkehr der Helligkeit der Ringfigur konnte ich hierbei nicht beobachten, wohl aber hat dieselbe Frau M. E. beobachtet, welche auf mein Ersuchen gleichfalls solche Versuche ausgeführt hat.

\section{Versuche bei geschlossenem Ange, in welches dnrch die Lider Licht fällt.}

Wenn ich unter solchen Verhältnissen das Auge stark nach innen gewendet habe, so erscheint die Ringfigur an der Aussenseite des blinden Fleckes, welche ich oben beschrieb, anders gefärbt und ganz besonders deutlich. Sie besteht aus einer dunkelblauen Scheibe, welche von einem hellen Kreise umgeben ist, der im blinden Fleck unterbrochen ist; dieser helle Kreis ist wieder von einem dunkelblauen Hof umgeben, der ohne scharfe Grenze in den rötlich-schwarzen Grund des Gesichtsfeldes übergeht. Dieses Phänomen haben bereits Purkinje, Czermak, Aubert und Helmholtz an den angeführten Orten in ähnlicher Weise beschrieben. Drücke ich aber zugleich in der beschriebenen Weise auf den Bulbus, so schrumpft dieses Phosphen im ganzen zusammen, wobei sich der helle Ring: derart gegen sein Zentrum verbreitert, dass von diesem nur mehr ein undeutlicher dunkler Fleck übrig bleibt. Der helle Kreisring erscheint nun hellgelb. Zugleich zeigt sich die der eingedrückten Stelle entsprechende Ringfigur als ein schwarzer Kreis mit hellem Zentrum und einem etwas matteren hellen Kreis an der Peripherie. Davon zieht ein keilförmiger schwarzer Fortsatz gegen den Fixations- 
punkt. Letzterer selbst erscheint von einem etwas dunkleren Kreise umgeben, welcher verwaschen in die Umgebung übergeht. Höre ich plötzlich auf zu drücken, so tritt an der Stelle der während des Druckes sichtbar gewordenen Ringfigur eine Umkehrung der Helligkeitsverhältnisse auf: es erscheint ein orangeroter Kreisring mit schwarzer Mitte, blitzartig auftretend und verschwindend. In der der Macula entsprechenden Gegend zeigt sich ein grosser purpurroter Kreis, in dessen Mitte am Fixationspunkte ein ganz hellgelbes Zentrum, umgeben von einem etwas dunkleren Kreise, verbleibt, welcher verwaschen in den purpurroten Kreis übergeht. An der Grenze der dem blinden Flecke angehörigen Stelle des Phosphens, welche früher, während des Druckes, gelbrot und heller als das übrige Gesichtsfeld erschien, tritt eine leichte Verdunklung auf, welche rasch wieder vorübergeht.

Von der Druckringfigur zieht an Stelle des früheren schwarzen Fortsatzes nun ein deutliches gelbes Bündel gegen den Fixationspunkt.

Dies sind meine Beobachtungen über das Druckphosphen unter den drei angegebenen Bedingungen.

Eine weitere Beobachtung sei hier noch erwähnt. Wenn ich die Augen geradeaus wendete, die Lider schloss, Sonnenlicht durch dieselben fallen liess, sodann irgendwo an der Peripherie einen ganz geringen Druck auf den Bulbus ausübte, so sah ich oftmals ausser dem beschriebenen Druckphosphen im Umkreis des Gesichtsfeldes einzelne kleinere Ringfiguren auftreten. Ich vermute nach ihrer Lage, dass sie den Ansatzstellen der Muskeln am Bulbus entsprechen.

Als Kontrollpersonen für meine Versuche habe ich mehrere Emmetrope, Ametrope und Aphake benutzt. Da die Beobachtung subjektiver Gesichtserscheinungen entschieden grosse Übung und Geduld, wohl auch persönliche Eignung erfordert, so sind nur selten brauchbare Beobachtungsresultate zu erhalten. Am wertvollsten sind offenbar die Beobachtungen völlig unbeeinflusster Personen. Überraschende Resultate, und zwar sofort auf den ersten Versuch, ergaben mir die Beobachtungen einer emmetropen Schweizerin und einer etwas myopen Tiroler Dame mit wenig prominenten Augen, der Frau Dr. Minna Epstein.

Letztere Beobachterin hat bei dem Druckversuch im Dunkeln auch die von Purkinje gezeichneten hellen und dunklen konzentrischen Bogen zwischen Ringfigur und Fixationspunkt gesehen, 
ohne überhaupt nur mit einem Wort über das, was zu sehen ist, verständigt worden zu sein. Sie hat mir diese Bogen genau so gezeichnet, wie sie Purkinje angibt.

Frau Dr. Epstein hat nun auch auf mein Ersuchen die Erscheinungen bei plötzlichem Aufhören des Druckes untersucht und mit vollster Sicherheit behauptet, jedesmal eine Umkehr der Helligkeitsverhältnisse der Ringfigur gesehen zu haben. Im übrigen stimmt ihre Beschreibung der Erscheinung mit der meinigen völlig überein.

Zwei Aphake, einen Mann und eine Frau mit extrahierter Katarakt, habe ich untersucht, um zu sehen, ob die Linse auf das Druckphosphen Einfluss nimmt. 'In beiden Fällen war bloss ein Auge der Linse beraubt, das andere noch im Besitze derselben, so dass ich an jeder Person vergleichsweise beide Augen untersuchen konnte.

In beiden Fällen wurde im Hellen bei offenem Auge und ebenso im Dunkeln ein schwarzer Kreis mit hellem Zentrum gesehen, so dass also kein Einfluss der Linse auf die Ringfigur zu eruieren ist.

Die Hauptsache erscheint es mir, festzustellen, dass ich und meine Kontrollpersonen den wichtigsten Bestandteil des primären Phosphens, die Ringfigur, stets mit einem hellen Zentrum und ferner im Dunkeln und im Hellen mit gleichen Helligkeitsverhältnissen sehen, was den Voraussetzungen widerspricht, auf denen Klein seine früher erwähnte Erklärung des Phosphens in Verbindung mit seiner neuen Theorie des Sehens durch das Selbstleuchten der Netzhaut aufbaut, und aus denen anderseits G. Schwarz den Schluss zieht, dass das Phosphen eine Folge des Gesetzes der Schwelle sei.

Für das Phosphen ist wohl hauptsächlich die Erklärung gebräuchlich, dass der schwarze Ring durch Druckanämie entstehe. Wenn nun diese Annahme an und für sich schon sehr wenig Wahrscheinlichkeit besitzt, so ist sie jedenfalls nicht imstande, die hellen Anteile des Phosphens zu erklären.

Ich habe mir über das Zustandekommen des Druckphosphens eine Meinung gebildet, welche ich im folgenden zu erörtern versuchen will.

Wenn schon der geringste Druck auf die Sklera, der ja noch durch die zwischen Sklera und dem drückenden Körper liegenden Weichteile abgeschwächt wird, das sehr komplizierte Druckphosphen 
zu erzeugen imstande ist, so erhellt daraus mit Sicherheit, dass ausserordentlich geringe, unmessbare Druckdifferenzen innerhalb der empfindlichen Elemente bereits zur Entstehung einer Gesichtsempfindung hinreichen.

Auf Grund dieser Erwägung schien es von Anfang an unwahrscheinlich, dass Formveränderungen als Ausdruck dieser Druckdifferenzen an der inneren Bulbuswand wabrzunehmen seien. Gleichwohl schien es mir unerlässlich, die Inspektion des Augenhintergrundes an der gedrückten Stelle nicht zu verabsäumen. Herr Professor Dimmer war so gütig, in seiner Grazer Klinik mein Auge, während ich mir das Druckphosphen erzeugte, zu spiegeln; es gelang aber nicht, die gedrïckte Stelle in den Bereich des Augenspiegels zu bringen.

Ich machte daher im Grazer physiologischen Institute folgenden Versuch: Ich eröffnete die laterale Wand der Orbita einer narkotisierten Katze, indem ich mit Schonung des Bulbus eine hinreichend grosse Öffnung in dieselbe machte, um zur'Rückseite des Bulbus zu gelangen. Nach Stillung der Blutung und Abtragung der hinderliehen Weichteile übte ich sodann mit dem Knopfe einer vorne abgebogenen Stahlsonde durch Hebelbewegungen derselben einen Druck auf die hintere Bulbuswand aus. Zu gleicher Zeit spiegelte Professor Dimmer diese nunmehr dem Spiegel leicht zugängliche Stelle des Katzenauges. Es trat sehr bald Trübung der Hornhaut durch Eintrocknung und Faltung ibres Epithels auf, welche das Spiegeln beeinträchtigte. An der gedrückten Stelle bemerkte Professor Dimmer bei gelindem Drucke keine Veränderung, erst bei starkem Drucke mit der Sonde trat eine blasse, blutleere Vorwölbung an der Innenseite der gedrückten Stelle auf. Dazu musste aber ein so heftiger Druck ausgeübt werden, wie man ihn am Menschenauge nie bewirken dürfte.

Faltungen waren in der Umgebung dieses Buckels nicht erkennbar. Dieses Experiment verlief also für den Erklärungsversuch des Druckphosphens resultatlos. Es blieb mir demnach für letzteren nur mehr der deduktive Weg übrig.

Dieser führte mich $\mathrm{zu}$ folgender Anschauung. Zuerst ist die Frage zu beantworten, welche Formveränderungen der Bulbus durch den Druck auf die Sklera erleidet. Diese Formveränderungen habe ich in Figur 2 darzustellen versueht. Durch den Druck im lateralen Augenwinkel wird der Bulbus gegen die mediale Orbitalwand ge- 
drückt.' (Wie ich eingangs zitierte, sah Elli ot bei starkem Drucke im lateralen Augenwinkel sogar ausser dem gewöhnlichen Druckphosphen noch einen zweiten Ring an der entgegengesetzten Seite, welcher von dem Drucke des Bulbus an die mediale Orbitalwand herrührte.) Da der Bulbus in der Richtung des Druckes nicht ausweichen kann, so wird seine Wandung an der gedrückten lateralen Seite abgeflacht (s. Fig. 2a). Der Grad dieser Abflachung hängt natürlich von dem des ausgeübten Druckes ab. Der unmittelbar gedrückte Anteil der Bulbuswand wird hierdurch unter einen grösseren Krümmungsradius gesetzt. Die notwendige Folge davon ist, dass in der Nachbarschaft dieser Abflachung infolge der Elastizität der Sklera einerseits und des intraokularen Druckes anderseits ein mehr oder

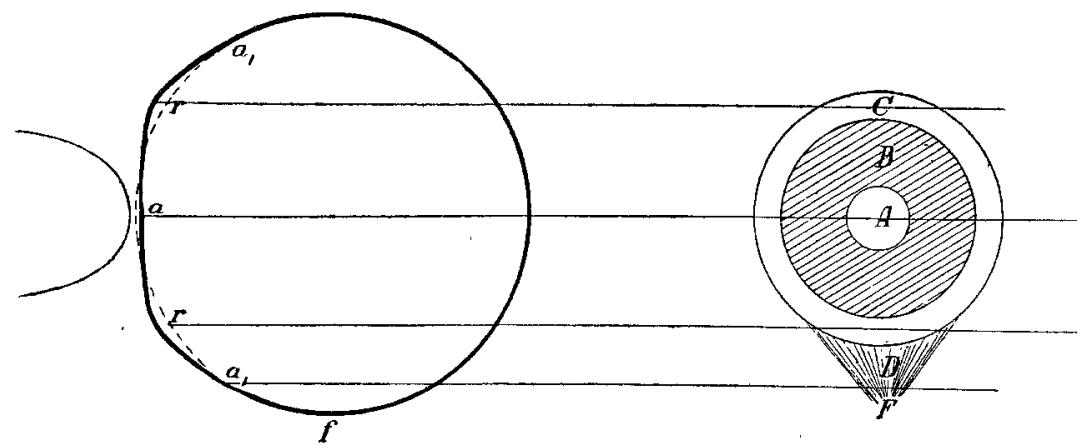

Fig. 2. Die Formveränderungen der Bulbuswand bei Druck auf die Sklera und das denselben entsprechende Phänomen. - a a, Abflachung. $r$ Ausbauchung. $f$ Fovea: $A$ helles Zentrum des Phosphens, $B$ dunkler Ring des Phosphens, $C$ heller Ring des Phosphens, $D$ dunkles Büschel des Phosphens, $F$ Fixationspunkt.

weniger allmählicher Übergang lieser Krümmung mit grösserem Radius zur normalen Krümmung stattfinden muss; dies kann nur dadurch geschehen, dass in der Nachbarschaft der Abflachung die Bulbuswand stärker gekrümmt, also unter einen kleineren Krümmungsradius gesetzt wird, als es die Norm ist. Dadurch entsteht eine ringförmige Ausbauchung der Bulbuswand in der Umgebung der Abflachung (Fig. 2, r). Dass dies so ist, davon kann man sich an jedem elastischen Ball, sei er mit Luft oder Wasser gefüllt, überzeugen. Die Abflachungsstelle liegt entweder symmetrisch oder asymmetrisch zum Bulbus, je nachdem der Druck in der Richtung des Radius oder einer von lateral vorn nach medial hinten ziehenden Sekante ausgeübt wurde. Je mehr der Druck nach rückwärts gerichtet ist, eine 
um so stärkere Abweichung von der normalen Krümmung wird die hintere Hälfte der Abflachungsstelle gegenüber der vorderen Hälfte derselben zeigen.

Welche Veränderungen gehen hierbei mit der inneren Schicht der Bulbuswand vor?

Netzhaut und Aderhaut spielen zufolge ihrer Weichheit, der lockeren Verbindung der Aderhaut mit der Sklera und der hierdurch bedingten Verschieblichkeit beider Häute ${ }^{1}$ ) nur eine passive Rolle; indem sie durch den intraokulären Druck an die Sklera angepresst werden und so die Formveränderungen dieser passiv mitmachen. Die Netzhaut, um die es sich punkto Deutung des Druckphosphens lediglich handelt, wird an der Stelle des ausgeübten Druckes abgeflacht, d. h. unter einen grösseren Krümmungsradius gesetzt, aber im Umkreise dieser Abflachungsstelle wird sie durch den intraokulären Druck an die ringförmige Ausbauchung der Sklera beim U̇bergang zur normalen Krümmung angedrückt und daher ebenfalls ausgebaucht, also unter einen kleineren Krümmungsradius gesetzt. Daraus folgt, dass die Netzhaut an der Stelle des Druckes entsprechend ihrer Abflachung unter gleichzeitiger Anpressung an die Sklera trotz der anscheinenden Verkleinerung der Strecke gezerrt (ihre Elemente somit voneinander entfernt), an der Stelle der Ausbauchung der Sklera im ringförmigen Übergangsteile trotz der anscheinenden Vergrösserung der Strecke zusammengedrückt wird, ihre Elemente also einander genähert werden. Dass dies so ist, kann man sich noch leichter veranschaulichen, wenn man sich die Krümmungsradien jeder Stelle der Bulbuswand zeichnet. Man wird finden, dass dieselben an der Stelle des Ausbauchungsringes stärker gegeneinander konvergieren als die Radien des normal gekrümmten Bulbus, an der Stelle der Abflachung aber weniger; die den inneren Anteilen dieser Radien (innerhalb der Bulbuswand) entsprechenden Netzhautelemente sind daher an der Abflachungsstelle voneinander entfernt, an der Ausbauchungsstelle einander genähert worden; mit anderen Worten: die Netzhaut hat der Fläche nach an der Stelle der Abflachung (Fig. $2 a$ ) eine Druckverminderung (Zerrung), an der Ausbauchungsstelle (Fig. $2 r$ ) eine Druckerhöhung (Kompression) erlitten.

1) Siehe: Ernst Fuchs, Über eine entoptische Erscheinung bei Bewegung des Augapfels. Arch. f. Ophthalm. 27. Jahrg. (3). 1881. 
Es fragt sich nun, welche Anteile dieser in Fig. 2 dargestellten Formveränderungen der Bulbuswand und welche Anteile des Phosphens einander zugehören. Entspricht die Abflachungsstelle (a) dem hellen Zentrum allein oder auch dem schwarzen Kreisring?

Folgendes Verhalten des Phosphens vermag vielleicht Aufschluss hierüber zu geben:

Bei ganz gelindem Drucke ist das helle Zentrum am grössten. Steigere ich den Druck, so wird es immer kleiner und schärfer begrenzt, hingegen wächst der schwarze Kreisring mit der Stärke des Druckes bis zu einer gewissen Grenze.

Bei stärkerem Drucke wird sicher die Abflachungsstelle vergrössert. Wenn also das helle Zentrum der Abflachung zukäme, so müsste es auch bei Vergrösserung dieser, also bei Steigerung des Druckes, wachsen. Statt dessen verbreitert sich aber unter letzterer Bedingung der schwarze Ring auf Kosten sowohl des hellen Zentrums als auch der Peripherie. Mit der Vergrösserung der $\mathbf{A b}$ flachung geht aber zugleich eine Vergrösserung des schwarzen Ringes einher. Daher glaube ich annehmen zu dürfen, dass die Abflachung: der Bulbuswand das Phosphen des schwarzen Kreisringes bedingt. Zugleich mit der Vergrösserung des letzteren schiebt sich auch der schmälere weisse Kreisring an dessen Peripherie weiter hinaus und wird um so heller und schärfer, je stärker der Druck ist (bis zu einer gewissen Grenze). Dies scheint mir sehr gut mit den angenommenen Formveränderungen, nämlich der ringförmigen Übergangsausbauchung, im Einklang zu stehen. Denn je stärker der Druck, um so stärker muss sich wohl auch diese ringförmige Ausbauchung ausbilden, und um so stärker wird dann infolge der Abnahme des Krümmungsradius die Retina an dieser Stelle komprimiert. Der dadurch bedingten Drucksteigerung innerhalb der lichtempfindlichen Schicht entspricht die Zunahme der Helligkeit und Deutlichkeit des weissen Ringes an der Peripherie.

Wie erklärt sich aber das helle Zentrum?

Eine Formveränderung der Bulbuswand während des Druckes, welche ihm entspräche, kenne ich nicht. Es bleibt im Gegenteile während des Fortschreitens der abgeleiteten Formveränderung zurück, ohne aber jemals ganz zu verschwinden. Es bleibt mir daher für seine Deutung nur die Annahme übrig, dass die Ursache des hellen Zentrums das direkte Aufliegen der harten, drückenden Fingerkuppe an dieser Stelle sei, also eine Drucksteigerung, eine Kom- 
pression in der Richtung des Druckes selbst (während ich für die anderen Teile des Phosphens Druckdifferenzen in der Richtung der Fläche der Bulbuswand verantwortlich mache). Diese Drueksteigerung in der Richtung des Druckes ist ja das allererste, welches die Formveränderungen, die Ursache der flächenhaften Druckdifferenzen, erst bedingt. Daher käme es, dass auch bei leisem Drucke, zuerst der helle Fleck auftritt, von dem bei Steigerung des Druckes das helle Zentrum des Phosphens übrigbleibt, indem durch jene die Abflachung und damit die Auseinanderzerrung der Retina bewirkt wird. Das helle Zentrum entspräche also, wenn der.Druck bereits zur Ausbildung des weissen und schwarzen Ringes genügte, jenem Anteil der an der Abflachungsstelle auseinandergezogenen lichtempfindlichen Elemente, welcher durch direktes Aufliegen auf dem drückenden Körper einem mechanischen Reize in der Druckrichtung ausgesetzt ist.

Die dunkle Umgebung des peripheren weissen Kreisringes, welche den Übergang zur Helligkeit des übrigen Gesichtsfeldes bildet, entspricht ihrer Lokalisation nach der an die ringförmige Ausbauchung. des Bulbus (Fig. 2r) angrenzenden Abflachung der Bulbuswand (Fig. $2 a_{1}$ ) mit ihrem allmählichen Übergange zur normalen Krümmung.

Der Vergleich der Formveränderungen der Bulbuswand während des auf die Sklera ausgeübten Druckes mit dem Phosphen ergibt aber, wenn wir annehmen dürfen, dass die Abflachungsstellen (Fig. $2 a$ und $a_{1}$ ) den dunklen Teilen, die ringförmige Ausbauchungsstelle $(r)$ dem hellen Ringe entsprechen, dass eine Druckverminderung der Netzhautfläche nach (Auseinanderziehung der lichtempfindlichen Elemente) die Empfindung „Dunkel“, eine Druckerhöhung (Kompression) die Empfindung „Hell“ erzeugt.

Ich will nun die Formveränderungen untersuchen, welche aller Wahrscheinlichkeit nach mit der Entstehung der anderen Teile des Druckphänomens einhergehen.

Die Erscheinung, welche an der Fixationsstelle eintritt, ist oft sehr kompliziert, sehachfigurenartig; dies entspricht wohl den Druckerscheinungen, welche man überhaupt bei Steigerung des intraokularen Druckes wahrnimmt; wie sie eingehend von Purkinje beschrieben worden sind ${ }^{1}$ ). Dass sie aber ausserdem noch in besonderer mechanischer Beziehung sowohl zur direkt gedrückten Stelle als auch zur

1) Purkinje, Beobachtungen und Versuche, I. :1819. 
Sehnerveneintrittsstelle stehen, beweist ihre Verbindung mit den Anteilen des Phosphens, welche diesen letzteren Stellen entsprechen, durch zwei dunkle Garben, welche sich vom Fixationspunkt aus

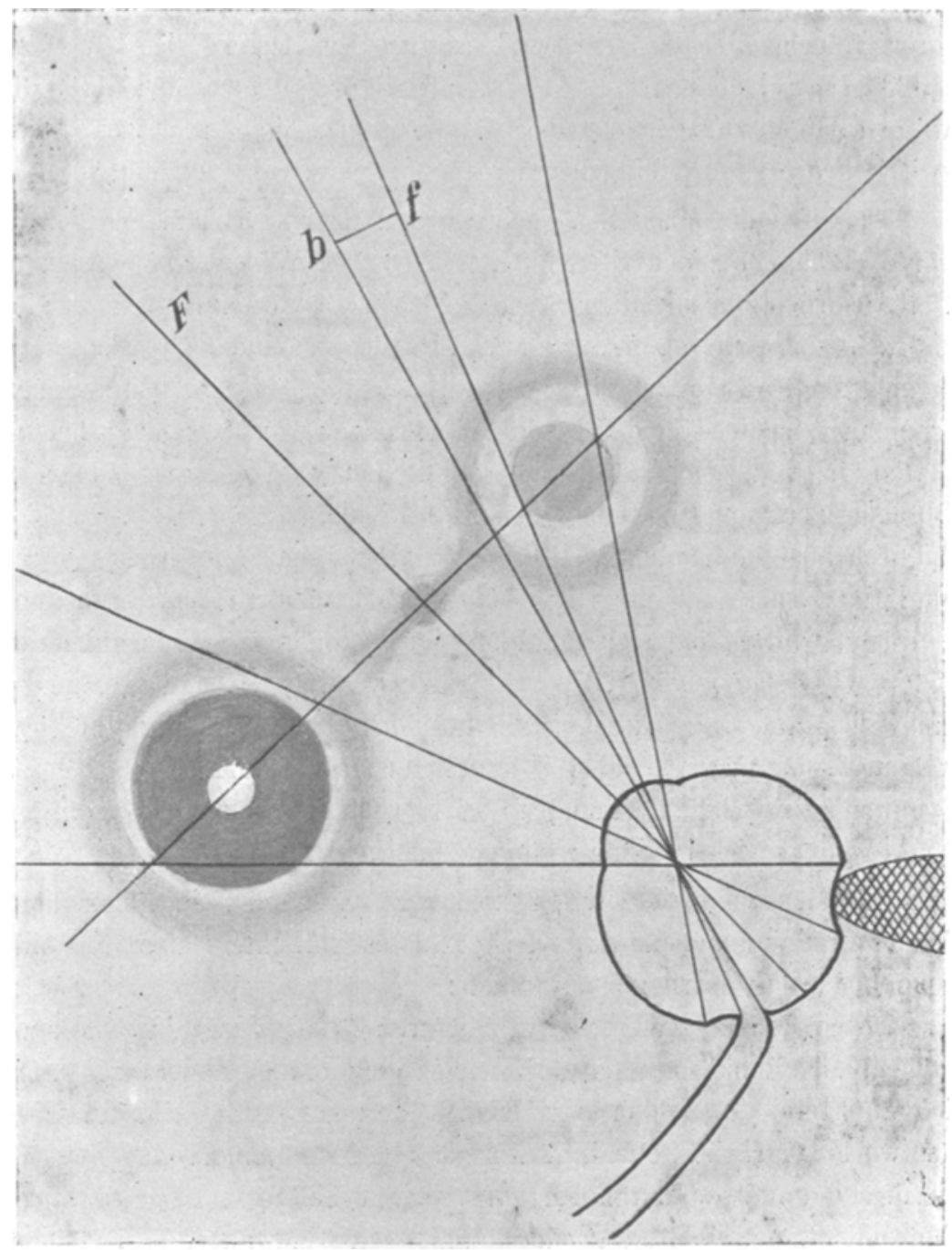

Fig. 3. Schematische Darstellung der Entstehung des Druckphosphens. $F$ Fixationspunkt. bf blinder Fleck.

gegen die Ringfigur einerseits und gegen den blinden Fleck anderseits verbreiteru und besonders bei stärkerem Drucke deutlich sichtbar werden, (s. Fig. 1 und 3). Die Einwärtswendung des Bulbus kann 
für die Entstehung dieser Garben nicht allein verantwortlich gemacht werden, weil sie anch bei geradeaus gerichtetem Bulbus auftreten, wenn man auf die Sklera drückt. Ich glaube, ihre Erklärung ergibt sich daraus, dass im lebenden Bulbus die Netzhaut in der Fovea ihrer Unterlage fester aufsitzt als an der Peripherie. Dies hat schon H. Müller behauptet und dafür als Grund angegeben, dass die wimperartigen Fortsätze des Pigmentepithels in der Fovea tiefer zwischen die Zapfen eindringen als in der übrigen Netzhaut ${ }^{1}$ ).

Daher setzt sich den Verschiebungen durch Druckdifferenzen in der Netzhaut an der Stelle der Fovea ein Widerstand entgegen. Der Ausdruck der hierdurch in der Richtung von der Fovea zur eingedrückten Stelle einerseits, zur Fixationsstelle der Netzhaut am Sehnerveneintritt anderseits zustande kommenden Druckdifferenzen scheinen mir diese beiden dunklen Büschel zu sein, welche zu beiden Seiten des Fixationspunktes auftreten. Ich glaube aber, dass das Büschel zwischen Fixationspunkt und Ringfigur an der Druckstelle dadurch zustande kommt, dass die Netzhaut, nachdem sie vom intraokularen Drucke in die ringförmige Ausbauchung (Fig. $2 r$ ) hineingedrängt worden ist, sich nun der hierauf folgenden Abflachung der Sklera (Fig. $2 a_{1}$ ) beim Übergang zur normalen Krümmung anlegen muss, wodurch die Netzhaut wieder einer Spannung ausgesetzt wird, welche in der Fovea einen Widerstand erfährt. Der Ausdruck dieser Spannung (zwischen $a_{1}$ und $f$ in Fig. 2) ist das von dem Fixationspunkte zur Druckringfigur ziehende dunkle Büschel (s. Fig. 3).

Das Büschel zwischen Fixationspunkt und blindem Flecke und das letzteren urngebende Phosphen scheinen mir ihre Entstehung folgenden Verhältnissen zu verdanken:

Durch den gesteigerten intraokularen Druck wird die Stelle, an welcher sich demselben der geringste Widerstand entgegensetzt, nämlich die Stelle des Sehnerveneintrittes mit der Lamina cribrosa, nach rückwärts gedrückt. Dadurch werden mit dem Sehnervenkopfe auch die diesem unmittelbar anliegenden Netzhautteile gegen den Skleroticochorioidealkanal gedrängt und damit ein $\mathrm{Zug}$ auf die Netzhaut im Umkreise derselben ausgeübt. Da dieser an der Fovea aus den oben erwähnten Gründen einen Widerstand findet, so wird die Netzhaut dadurch zwischen Sehnervenkopf und Fovea gespannt, und die dadurch entstehende Druckverminderung zwischen den betroffenen

1) H. Müller, Gesammelte Schriften, herausg. von Becker. 1872. 
lichtempfindlichen Elementen erzeugt das dunkle Dreieck zwischen Fixationspunkt und blindem Flecke.

Dieser Spannung der Netzhaut an ihrer Ansatzstelle am Skleroticochorioidealkanale entspricht auch der undeutliche Fleck, welcher bei geradeaus gerichtetem Blicke durch Druck auf die Sklera entsteht.

Wenn gleichzeitig während dieses Druckes das Auge adduziert wird, so übt dabei der Sehnerv einen Zug auf die vom Sehnervenkopf nach aussen, also gegen die Fovea zu gelegene Partie der Netzbaut aus, welcher die früher erwähnte Spannung und damit auch das ihr entsprechende dunkle Büschel verstärkt. Anđerseits aber wird durch den Sehnervenzug bei der Adduktion des Bulbus an der medialen Seite des Sehnerveneintrittes die Netzhaut zusammengeschoben, ja sogar wahrscheinlich, wie Helmholtz ${ }^{1}$ ) vermutet, die Sklera etwas eingebogen (siehe Fig. 3); dadurch kommen hier ganz ähnliche Formveränderungen in der dem Sehnervenkopf medial anliegenden Bulbuswand zustande wie an der Stelle des von aussen auf den Bulbus ausgeühten Druckes: die Netzhaut erleidet medial von der Papille eine Impression, welche mit zwei allmählich verlaufenden Schenkeln die Papille oben und unten umfängt; im Umkreise dieser Impression findet aus den gleichen Gründen wie bei der durch den Druck erzeugten Abflachung eine ringförmige Kompression der Netzhaut (Druckerhöhung) statt, während im Gebiete der Impression selbst eine Auseinanderziehung der Netzhautelemente (Druckverminderung) eintritt.

Diese mechanische Veränderung ruft das Ringphosphen hervor, welches in der Gegend des blinden Fleckes und auswärts von diesem schon bei blosser starker Adduktion des Bulbus ohne Druck sichtbar wird ${ }^{2}$ ) (s. Fig. 3). Der dunkle Fleck in der Mitte des hierbei am blinden Flecke sichtbaren Phänomens, welchen gleich Purkinje, A ubert und $\mathrm{Helmholtz}$ ) auch ich sehe, erklärt sich aus der Auseinanderziehung (Spannung) der Netzhaut im Gebiete ihrer Ein-

1) Physiol. Optik, II. Auf., S. 727.

2) Bei der Abduktion des Bulbus tritt das Phänomen, wie Helmholtz beobachtete (Physiol. Optik S. 727), zwischen Fixationspunkt und blinden Fleck, also einwärts von diesem, auf, was die hier gegebene mechanische Ableitung dieses Phänomens stützt; denn hier tritt die Einbuchtung der Netzhaut auswärts von der Papille auf.

3) Siehe die eingangs erwähnten Zitate. 
buchtung, der sehr deutliche helle Ring, welcher den dunklen Fleck umgibt, aus der Kompression der Netzhaut rings um die Einbuchtung.

Übe ich zugleich während der Einwärtswendung einen Druck auf den Bulbus aus, so verbreitert sich der helle Ring auf Kosten seines dunklen Zentrums, ja dieses kann unter Umständen gänzlich verschwinden, so dass nur ein heller Kreis in der Gegend des blinden Fleckes sichtbar bleibt. Die Erklärung dieser Veränderung ergibt sich möglicherweise aus der Kompression der gegen das Bulbusinnere vorgebuchteten Netzhautpartie durch den auf diese wirkenden fortgepflanzten Druck. Auch Purkinje hat, wie ich eingangs zitierte, bei diesem Versuche einen Lichtkreis in der Gegend der Papille beobachtet.

Die von Purkinje beschriebenen und von E. Fuchs ${ }^{1}$ ) und ebenso von einer meiner Kontrollpersonen, Frau Wilhelmine Epstein, beobachteten konzentrischen Streifen, welche am fovealen Rande des der Druckstelle entsprechenden Ringphosphens bei möglichst nach rückwärts gerichtetem Drucke auf die Sklera auftreten, finden ihre Erklärung vielleicht darin, dass zufolge der nach rückwärts zielenden Druckrichtung in den hinten an die Abflachungsstelle angrenzenden Teilen der Aderhaut und Netzhaut nach vorne konkave bogenförmige Druckdifferenzen oder im extremen Falle sogar Falten entstehen. Auf der Höhe dieser Falten fände dann eine Spannung der inneren Oberfläche, also eine Druckverminderung innerhalb der Netzhaut parallel zur Oberfläche, in den Faltentälern eine Zusammenschiebung der inneren Teile der Bulbuswand, also eine Zusammendrückung der lichtempfindlichen Elemente statt. Nach den Anschauungen, welche ich aus dem Verhalten der übrigen Teile des Phosphens abgeleitet habe, entsprächen den Faltenhöhen die dunklen, den Faltentälern die hellen konzentrischen Bogen zwischen Ringphosphen und Fixationsstelle.

Diese Anschauung scheint mir dadurch an Wahrscheinlichkeit zu gewinnen, dass sich mit ihrer Hilfe ähnliche Phänomene zwanglos erklären lassen.

Ein solches Phänomen sind die feinen konzentrisehen, abwechselnd lichten und dunklen Streifen, welche Purkinje ${ }^{2}$ ) bei Seitwärtswendung des Auges an der gegen den Mittelpunkt des Sehfeldes ge-

1) $1 . \mathrm{c}$.

2) Beobachtungen und Versuche, I S. 80. 
legenen Seite des blinden Fleckes beobachtete, deren Ähnlichkeit mit den beim Druckphosphen gesehenen er selbst ausdrücklich hervorhob ${ }^{\mathbf{1}}$ ). Bei der forcierten Abduktion des Bulbus ist es nun leicht möglich, dass die Netzhaut an der Aussenseite der Papille in feine zirkuläre Falten gelegt wird, deren Bergen die dunklen und deren Tälern die hellen Bogen meiner Ableitung gemäss entsprechen, resp. dass dementsprechende bogenförmige Druckdifferenzen entstehen. Ein ähnliches Phänomen hat E. Fu chs ${ }^{2}$ ) beschrieben. Bei starker Auswärtswendung des Auges sah er auf einem neben den Kopf gehaltenen weissen Papier einen grossen farbigen Ring und an dessen nasaler Seite, in den Ring eingetragen, zwei Systeme von übereinander geschichteten konzentrischen krummen Linien, welche, durch den Fixationspunkt gehend, sich verdoppeln, dabei aber lichtschwächer werden.

Fuchs erklärt dieses Phänomen in folgender Weise: „Bei sehr rascher Auswärtswendung des Auges wird der hintere Pol desselben vehement nach innen gedreht. Dies kann nicht ohne Zerrung des Opticus abgehen, welche sich in die unmittelbar umgebende Netzhaut fortpflanzt. Die Erschütterung der letzteren gibt sich als Lichtring zu erkennen. In der Gegend des hinteren Poles, wo die Netzhaut besonders dünn ist, kommt es sogar zu feinen Faltungen der Netzhaut, welche entoptisch als bogenförmige Linien wabrnehmbar werden."

Von dem Lichtring seines Phänomens glaubt Fuchs, „dass derselbe der Ausdruck einer den Opticus kreisförmig umgebenden Welle sei, welche an der äusseren Seite des Opticus einer Verdichtung, an der inneren Seite einer Verdünnung (Zerrung) der Netzhaut entspricht".

In dem den Bogenlinien entsprechenden Anteil des Gesichtsfeldes beobachtete Fuchs Metamorphopsie, indem die durch die Bogenfigur gesehenen Gegenstände „leicht unregelmässig, wie gewellt" erschienen. Daraus bekam Fuchs den Eindruck, dass es sich hier um Netzhautfalten handle. Fuchs berechnet die Höhe dieser Netzhautfalten, ohne jedoch die Frage im besonderen zu erörtern, welchem Anteil derselben die hellen und welehem die dunklen Ringe entsprechen.

1) Beobachtungen und Versuche, I S. 138.

2) E. Fuchs, Uber eine entoptische Erscheinung bei Bewegung des Augapfels. Arch. f. Ophthalm. 27. Jahrg. (3) S. 33 ff. 1881. 
Beim Aufhören des Druckes tritt bei dem von mir beobachteten Druckphospheu eine Umkehr der Helligkeits- und Farbenverhältnisse auf, und zwar unter allen Bedingungen der Beobachtung, wie ich ja eingehend beschrieben habe. Die Umkehr der Farbenverhältnisse zeigt sich namentlich bei dem Versuche mit geschlossenen Lidern, durch welche Licht ins Auge fällt, deutlich. In rudimentärer Weise ist diese Beobachtung eines "negativen Nachbildes" des Druckphosphens, welche ich für alle Einzelheiten desselben mit absoluter Sicherheit gemacht habe, auch sehon von Purkinje und Aubert an den von mir zitierten Stellen beschrieben worden.

Eine Umkehr der Helligkeitsverhältnisse bei abwechselnder Projektion auf hellen und dunklen Grund oder beim Blinzeln, wie sie negativen Nachbildern nach Lichteindrücken zukommt, habe ich schon deshalb nicht beobachten können, weil diese Erscheinungen dazu zu flüchtig sind.

Da man gewöhnt ist, von Nachbildern nur bei adäquater Reizung des Auges zu sprechen, so erscheint es mir nicht ohne weiteres statthaft, die gleiche Bezeichnung bei mechanischen und elektrischen Reizen zu gebrauchen. Jedoch betone ich hier die grosse Ähnlichkeit der Umkehr der Farben- und Helligkeitsverhältnisse der durch mechanischen Reiz erzeugten Phänomene nach Aufhören dieses Reizes mit dem negativen Nachbilde nach Lichtreiz. Jenes "negative Nachbild" lässt sich, glaube ich, in naheliegender Weise dadurch erklären, dass beim Aufhören des ausgeübten Druckes infolge der Elastizität der beteiligten Elemente eine sofortige Rückkehr der verschobenen Elemente zu ihrer Gleichgewichtslage und damit zugleich eine relative Umkehr der Druckverhältnisse eintritt. Letztere scheint mir zur Erklärung der Umkehr der Helligkeitsverhältnisse des Druckphänomenes nach Aufhören des Druckes hinreichend. Zu diesem mechanischen Reize der Rückkehr zur Gleichgewichtslage kommt aber noch der innere Reiz, welcher durch die Rückkehr der lichtempfindlichen Elemente aus dem Zustande der Erregung in den der Ruhe erzeugt wird, welch letzteres vielleicht eine Ursache der Nachbilder nach kurzdanernden Lichteindrücken darstellt.

$\mathrm{Zu}$ beantworten ist noch die Frage, in welchem Teile der Netzhaut die mechanische Reizung wirksam wird. Gad und Heymans ${ }^{\mathbf{1}}$ ) schreiben: „Ob der Nageldruck auf Endapparat oder Fasern wirkt, ist ungewiss, doch scheint wegen der Figur der Druckerscheinung

1) Gad-Heymans, Lehrbuch d. Physiol. d. Menschen 1892 Ș. 202. 
ersteres wahrscheinlich." Mir scheint es zufolge der deutlichen Form des Phosphens ausgeschlossen, dass die primäre Erregung desselben in der Nervenfaserschicht zustande käme. Zur weiteren Untersuchung dieser Frage schiene mir die mechanische Reizung solcher peripherer Stellen geeignet, in deren Gebiet die musivische Schicht durch pathologische Verbältnisse funlationsunfähig geworden ist, während die darüberziehende Nervenfasersehicht dadurch als funktionierend erkannt wird, dass peripher von dieser Stelle noch gesehen wird. Ich balte also Fälle von Retinochorioiditiden mit ausgebreiteten Skotomen, von welchen peripher noch Lichtempfindung besteht, für geeignet zur Untersuchung des Ortes der mechanischen Reizung.

Ich habe bisher erst einen derartigen Fall daraufhin untersuchen können; und in diesem war die genaue Lokalisierung durch Hornhauttrübung vereitelt, so dass ich aus den Resultaten keine Schlüsse ziehen kann.

Die wesentlichen Ergebnisse der vorliegenden Mitteilung sind einerseits solche, welche sich auf das Druckphosphen allein, anderseits solche, welche sich auf die Theorie der Gesichtswahrnehmung beziehen; in kurzen Worten folgende:

A. Bezüglich des Druckphosphens:

1. die der gedrückten Stelle entsprechende Ringfigur zeigt bei mir und meinen Mitbeobachtern stets ein helles Zentrum;

2. das Druckphosphen ist im Hellen und im Dunkeln nur quantitativ, nicht qualitativ durch Helligkeits- und Farbenverhältnisse unterschieden;

3. beim Aufhören des Druckes erscheint unter allen Bedingungen eine Umkehr der Helligkeits- und Farbenverhältnisse aller Teile des Druckphosphens;

4. der Ort der mechanischen Reizung ist aller Wahrscheinlichkeit nach nicht die Nervenfaserschicht;

5. die dunklen Partien des Druckphosphens entsprechen solchen Stellen der Netzhaut, welche sich im Zustande einer Druckverminderung (Zerrung), die hellen Anteile des Phänomens solchen, welche sich im Zustande einer Druckerhöhung (Kompression) befinden.

B. Allgemeine Schlüsse:

1. Druckerhöhung, Kompression der empfindlichen Netzhautelemente ruft Helligkeits-, Druckverminderung, Zerrung derselben Elemente Dunkelheitsempfindung hervor;

E. Pflüger, Archiv für Physiologie. Bd. 115. 
272 Robert Stigler: Beiträge zur Kenntnis des Druckphosphens.

2. plötzliche Änderung der Druckdifferenzen (während der Rückkehr zur Ruhelage) wirkt als Reiz und erzeugt ein Phänomen analog dem negativen Nachbilde.

Zum Schlusse gereicht es mir zur angenehmen Pflicht, Herrn Hofrat Sigmund Fxner für die Anregung und Förderung der vorliegenden Arbeit meinen ergebensten Dank auszusprechen. Ausserdem danke ich ergebenst Herrn Professor Dimmer für die freundlichst übernommene ophthalmoskopische Untersuchung der gedrückten Bulbi. 